\title{
Hernie diaphragmatique massive par élargissement de l'orifice hiatal postopératoire : une complication rare de l'œsophagectomie
}

\section{Massive Diaphragmatic Hernia Following Surgical Esophageal Hiatus Dilatation: an Unusual Complication of Oesophagectomy}

\author{
P. Catoire · M. Dubourg · G. Evrard
}

Reçu le 10 avril 2018; accepté le 30 mai 2018

(C) SFMU et Lavoisier SAS 2018

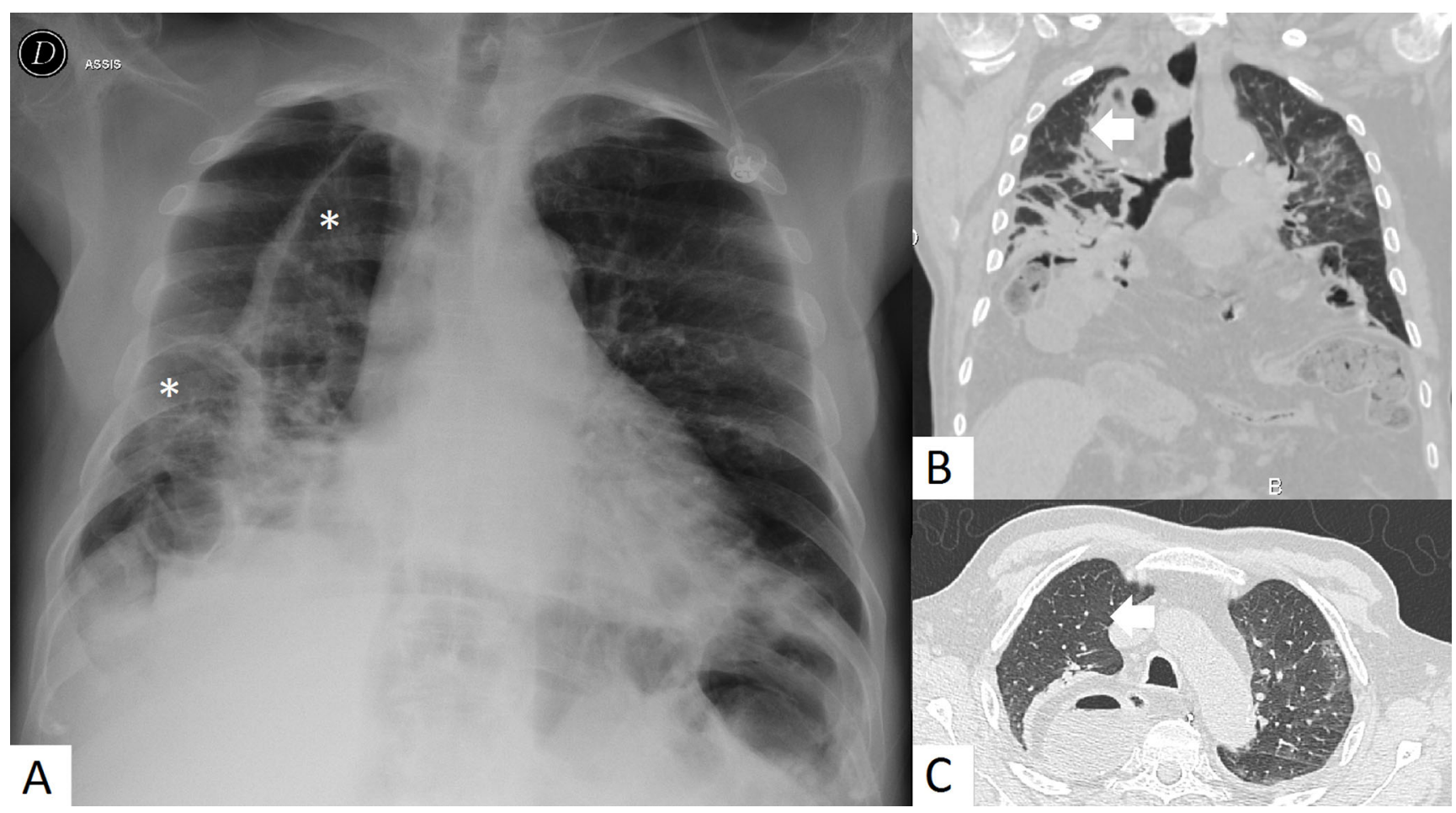

Fig. 1 Radiographie thoracique de face (A), tomodensitométrie thoracique en coupe coronale (B) et transversale (C). A. Hyperclartés (astérisques) témoignant d'une ascension des anses digestives. B et C. TDM montrant le refoulement du poumon droit vers l'avant et le dehors (flèches blanches)

Un patient de 84 ans se présente aux urgences pour un arrêt de transit depuis six jours, avec douleur abdominale diffuse progressive, sans vomissement ni fièvre. Il a bénéficié sept mois plus tôt d'une œesophagectomie par technique de

P. Catoire · M. Dubourg · G. Evrard ( $₫)$

Service d'accueil des urgences adultes,

groupe hospitalier Pellegrin,

centre hospitalier et universitaire de Bordeaux,

place Amélie-Raba-Léon, F-33000 Bordeaux, France

e-mail : gregoire.evrard@chu-bordeaux.fr
Lewis-Santi pour adénocarcinome du tiers inférieur de l'œsophage. Il ne présente pas de signe de détresse hémodynamique ou respiratoire à l'arrivée. L'examen abdominal retrouve une sensibilité diffuse sans contracture et une raréfaction des bruits hydroaériques abdominaux. L'auscultation retrouve une hypoventilation alvéolaire avec des bruits hydroaériques perçus jusqu'au tiers supérieur du champ pulmonaire droit. Le reste de l'examen est sans particularité. La radiographie thoracique (Fig. 1) retrouve une élévation des anses digestives en regard de l'hémichamp pulmonaire droit en lien avec une hernie diaphragmatique 
massive. Le bilan est complété par une tomodensitométrie abdominopelvienne réalisée après injection permettant de confirmer la hernie, avec engagement partiel du côlon droit et transverse ainsi qu'une partie des anses grêliques, sans signe de souffrance pariétale. Ce cas correspond à un syndrome occlusif bas par hernie diaphragmatique avec étranglement herniaire. La prise en charge opératoire réalisée le jour même permet une réintégration des anses digestives sans résection ainsi qu'une fermeture de l'orifice herniaire. Le patient quitte le service de chirurgie sept jours plus tard, sans complication notable.

La hernie diaphragmatique est une complication rare de l'œsophagectomie, avec une incidence estimée entre 1 et $3 \%$ et une morbimortalité importante. Les facteurs de risque identifiés incluent l'obésité et la localisation de la tumeur au cardia, sans prédominance significative de l'âge [1]. Les formes symptomatiques représentent $70 \%$ des cas et associent douleur abdominale, nausées, vomissements, signes d'obstruction et rarement dyspnée. La radiographie pulmonaire montre une opacité à base inférieure avec parfois mise en évidence d'un niveau hydroaérique. La confirmation diagnostique par tomodensitométrie permet la recherche de compli- cations et notamment de signes de souffrance digestive pariétale ainsi que l'élimination de diagnostics différentiels (abcès pulmonaire, diverticule épiphrénique). La prise en charge chirurgicale comprend une réintégration des anses digestives, une fermeture de l'orifice herniaire au besoin à l'aide d'une plaque et si nécessaire une colectomie en présence de lésions ischémiques [2]. La mortalité postopératoire est estimée entre 8 et $20 \%$. En conclusion, malgré sa relative rareté, l'étranglement d'anses digestives au sein d'un orifice herniaire hiatal nécessite un traitement rapide et doit être retenu comme diagnostic différentiel d'un syndrome occlusif, en particulier en cas d'antécédent de chirurgie œsophagienne.

\section{Références}

1. Adreou A, Pesthy S, Struecker B, et al (2017) Incidence and risk factors of symptomatic hiatal hernia following resection for gastric and esophageal cancer. Anticancer Res 37:7031-6

2. Audebert A, Wind P, Sauvanet A, et al (2005) Caractéristiques des hernies diaphragmatiques après œsophagectomie pour cancer. Ann Chir 130:21-5 